\title{
Colonoscopy in France during the COVID-19 pandemic
}

\author{
Antoine Meyer ${ }^{1,2,3}$ (D) Jérôme Drouin ${ }^{1}$ (D) $\cdot$ Mahmoud Zureik ${ }^{1,4} \cdot$ Alain Weill $^{1}$ (D) $\cdot$ Rosemary Dray-Spira $^{1}$ (i)
}

Accepted: 11 December 2020 / Published online: 7 January 2021

(C) The Author(s), under exclusive licence to Springer-Verlag GmbH, DE part of Springer Nature 2021

\begin{abstract}
Purpose We hypothesized that the COVID-19 pandemic may have modified dispensing of colonoscopy preparations, a proxy for the number of colonoscopies performed. We therefore studied changes in dispensing of colonoscopy preparations during the pandemic in France.

Methods Using the French national health data system, we identified colonoscopy preparations dispensed from 2018 to 2020. The expected 2020 dispensations were estimated from 2018 to 2019 dispensations.

Results Dispensing of colonoscopy preparations decreased markedly during the eight weeks of national lockdown: 83,045 colonoscopy preparations were dispensed, i.e., 181,826 (68.6\%) fewer than expected. After lockdown, dispensing of colonoscopy preparations gradually returned to expected numbers. Overall, this represents an estimated decrease of roughly 250,000 colonoscopy preparations during the six-month period following onset of the pandemic. This shortfall in the dispensing of colonoscopy preparations was of the same order of magnitude in people under or over 50 years of age, in men and women, and in those in the highest and the lowest quintiles of the deprivation index.

Conclusion In conclusion, roughly 250,000 fewer colonoscopy preparations were dispensed during the first six months of the COVID-19 pandemic in France. Deleterious consequences on morbidity and mortality related to gastroenterological diseases, such as colorectal cancer, are to be feared.
\end{abstract}

Keywords Colonoscopy $\cdot$ COVID-19 $\cdot$ Colorectal cancer $\cdot$ France

\section{Background and aims}

The COVID-19 pandemic emerged in France in February 2020. A national population lockdown restricted human contacts and travel to a strict minimum from March 17, 2020, until May 11, 2020. After lockdown, human interactions with protective measures were subsequently resumed. Many patients experienced difficulties or fear of accessing health care

Antoine Meyer

antoine.meyer@aphp.fr

Mahmoud Zureik

https://orcid.org/0000-0002-8393-4217

1 GIS-EPIPHARE, Épidémiologie des produits de santé, ANSM-CNAM, 42 bd de la Libération, 93200 Saint Denis, France

2 Assistance Publique-Hôpitaux de Paris, Hôpital Bicêtre, 78 Rue du Général Leclerc, 94270 Le Kremlin Bicêtre, France

3 Université Paris Sud, Le Kremlin Bicêtre, France

4 Université Versailles Saint-Quentin-en-Yvelines, Montigny-le-Bretonneux, France during and after lockdown [1]. We hypothesized that the COVID-19 pandemic may have modified dispensing of colonoscopy preparations, a proxy for the number of colonoscopies performed. We therefore studied changes in dispensing of colonoscopy preparations during the pandemic in France.

\section{Materials and methods}

This study was conducted using the French national health data system (Système National des Données de Santé, SNDS), which provides comprehensive healthcare claims data for $99 \%$ of the French population. The number of colonoscopy preparations dispensed by retail pharmacies was assessed by two-week periods for the first 37 weeks of 2018, 2019, and 2020. Colonoscopy preparations included polyethylene glycol, phosphate, or magnesium preparations. The study focused on the four weeks preceding lockdown (February 17 to March 15, 2020), the eight weeks of lockdown (March 16 to May 10, 2020), and the eighteen weeks following unlocking (May 11 to September 13, 2020). Expected numbers of users during these periods were estimated from numbers of users 
recorded during the corresponding weeks in 2018-2019: We applied a ratio of 1.0155 (number of users in 2020/mean number of users in 2018-2019) estimated between January 6 and February 16 to account for the increased number of prescriptions over calendar years. Differences between observed and expected numbers of colonoscopy preparations were computed by two-week periods, overall, and by sex, age, and area of residence deprivation index categorized in quintiles [2].

\section{Results}

During the four weeks preceding lockdown, the number of colonoscopy preparations dispensed was slightly higher than expected $(+2.1 \%)$ (Fig. 1 and Appendix Table 1). During the eight weeks of lockdown, dispensing of colonoscopy preparations decreased markedly by up to $85.6 \%$ between March 30 and April 12 compared to expected numbers. Overall, during lockdown, 83,045 colonoscopy preparations were actually dispensed, i.e., 181,826 (68.6\%) fewer than expected. During the four months after lockdown, the number of colonoscopy preparations dispensed remained lower than expected, but gradually returned to expected levels, resulting in 73,761 (13.9\%) fewer dispensings than expected. During the six-month period following onset of the pandemic, 255,587 fewer colonoscopy preparations than expected were dispensed. This shortfall in the dispensing of colonoscopy preparations was of the same order of magnitude in people under or over 50 years of age, in men and women, and in those in the highest and the lowest quintiles of the deprivation index.

\section{Discussion}

This study highlights a marked decrease of up to $85.6 \%$ in the dispensing of colonoscopy preparations during lockdown in France, which was not compensated after unlocking, corresponding to an estimated decrease of roughly 250,000 colonoscopy preparations during the six-month period following onset of the COVID-19 pandemic. These figures must be interpreted in the light of the estimated 1,000,000 colonoscopy preparations performed each year in France under normal conditions [3].

The effectiveness of colorectal cancer screening has been well demonstrated [4]. Indeed, the effectiveness of fecal occult blood test, as part of a two-stage screening strategy, depends on adequate follow-up colonoscopy. It has been observed that each month prior to colonoscopy is associated with an increased risk of colorectal cancer in an advanced stage and mortality [5]. Delayed surveillance colonoscopy for patients with inflammatory bowel disease could also increase the risk of high-grade dysplasia and colorectal cancer [6]. The decreased number of colonoscopies performed during the COVID-19 pandemic can therefore be expected to be associated with an increased colorectal cancer mortality. It can also be hypothesized that delaying colonoscopy in patients with inflammatory bowel disease, such as for the prevention of postoperative recurrence in Crohn's disease, may lead to more delayed detection of recurrences, making it more difficult to maintain clinical remission [7].

Limitations of this study include the following: the use of a proxy, i.e., dispensing of a colonoscopy

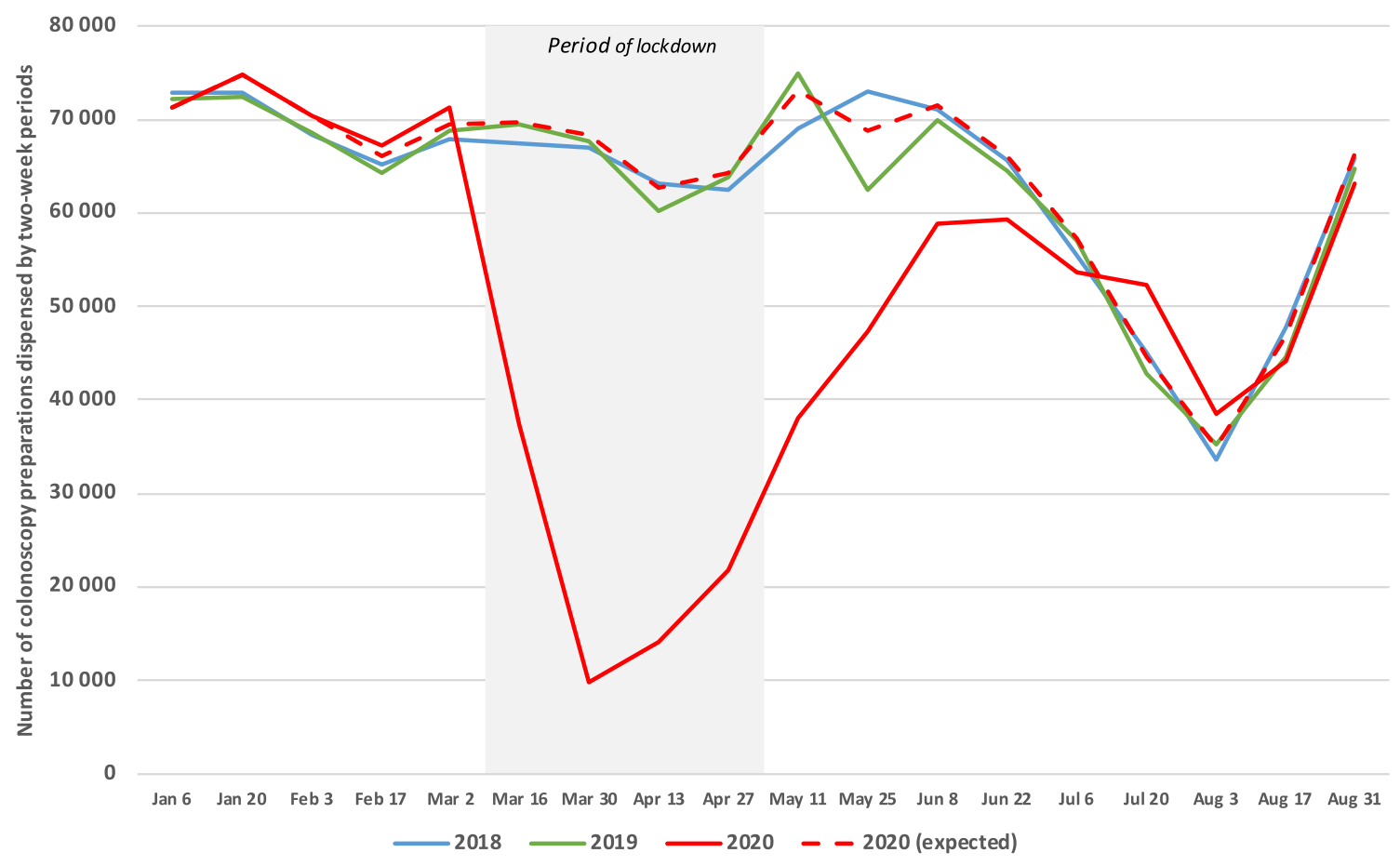

Fig. 1 Numbers of colonoscopy preparations dispensed in France by two-week periods in 2018, 2019, and 2020 (observed and expected) 
preparation, to estimate the number of colonoscopies performed, and the lack of data on hospital dispensing of colonoscopy preparations, i.e., colonoscopy preparations performed in hospital (less than $10 \%$ of all colonoscopy preparations [8]).

In conclusion, roughly 250,000 fewer colonoscopy preparations were dispensed during the first six months of the COVID-19 pandemic in France. Deleterious consequences on morbidity and mortality related to gastroenterological diseases, such as colorectal cancer, are to be feared. Ensuring continuity of gastroenterological care should be a priority in the context of current and future epidemics.

Supplementary Information The online version contains supplementary material available at https://doi.org/10.1007/s00384-020-03816-3.

Acknowledgments The authors would like to thank Anthony Saul, MD, for the assistance with English grammar and spelling.

Authors' contributions Study concept: Mahmoud Zureik, Alain Weill, and Rosemary Dray-Spira. Acquisition of data: Jérôme Drouin. Statistical analysis, interpretation of data, and drafting of the manuscript: Antoine Meyer. Critical revision of the manuscript for important intellectual content: every author. Study guarantor: Rosemary Dray-Spira.

\section{Compliance with ethical standards}

Conflict of interest The authors declare that they have no conflict of interest.

Ethical approval The French public institution that conducted this study has permanent access to the SNDS database in application of the provisions of Articles R. 1461-12 et seq. of the French Public Health Code and the French data protection authority decision CNIL-2016-316.

Informed consent Informed consent was therefore not required.

\section{References}

1. Weill A, Drouin J, Desplas D, et al (2020) Covid-19 : usage des médicaments de ville en France. EPI-PHARE. Available at: https:// www.epi-phare.fr/rapports-detudes-et-publications/covid-19-usagedes-medicaments-de-ville-en-france-rapport4/. Accessed 18 Oct 2020

2. Rey G, Jougla E, Fouillet A, Hémon D (2009) Ecological association between a deprivation index and mortality in France over the period 1997 - 2001: variations with spatial scale, degree of urbanicity, age, gender and cause of death. BMC Public Health 9:33

3. Blotière P-O, Weill A, Ricordeau P, Alla F, Allemand H (2014) Perforations and haemorrhages after colonoscopy in 2010: a study based on comprehensive French health insurance data (SNIIRAM). Clin Res Hepatol Gastroenterol 38:112-117

4. Schreuders EH, Ruco A, Rabeneck L, Schoen RE, Sung JJY, Young GP, Kuipers EJ (2015) Colorectal cancer screening: a global overview of existing programmes. Gut 64:1637-1649

5. Corley DA, Jensen CD, Quinn VP, Doubeni CA, Zauber AG, Lee JK, Schottinger JE, Marks AR, Zhao WK, Ghai NR, Lee AT, Contreras R, Quesenberry CP, Fireman BH, Levin TR (2017) Association between time to colonoscopy after a positive fecal test result and risk of colorectal cancer and cancer stage at diagnosis. JAMA 317:1631-1641

6. Cairns SR, Scholefield JH, Steele RJ, Dunlop MG, Thomas HJ, Evans GD, Eaden JA, Rutter MD, Atkin WP, Saunders BP, Lucassen A, Jenkins P, Fairclough PD, Woodhouse CR, British Society of Gastroenterology, Association of Coloproctology for Great Britain and Ireland (2010) Guidelines for colorectal cancer screening and surveillance in moderate and high risk groups (update from 2002). Gut 59:666-689

7. Iacucci M, Cannatelli R, Labarile N, Mao R, Panaccione R, Danese S, Kochhar GS, Ghosh S, Shen B (2020) Endoscopy in inflammatory bowel diseases during the COVID-19 pandemic and post-pandemic period. Lancet Gastroenterol Hepatol 5:598-606

8. Laanani M, Weill A, Carbonnel F, Pouchot J, Coste J (2020) Incidence of and risk factors for systemic adverse events after screening or primary diagnostic colonoscopy: a nationwide cohort study. Am J Gastroenterol 115:537-547

Publisher's note Springer Nature remains neutral with regard to jurisdictional claims in published maps and institutional affiliations. 